\title{
BOOLEAN ALGEBRAS WITHOUT NONTRIVIAL ONTO ENDOMORPHISMS EXIST IN EVERY UNCOUNTABLE CARDINALITY
}

\author{
JAMES LOATS ${ }^{1}$ AND MATATYAHU RUBIN
}

\begin{abstract}
We prove, assuming ZFC, that for every uncountable cardinal $\lambda$, there is a Boolean algebra of cardinality $\lambda$, without onto endomorphisms other than the identity.
\end{abstract}

1. Introduction. In this note we construct Boolean algebras (hereafter denoted by BA's), in order to prove the following theorem.

THEOREM 1.1. For every uncountable cardinal $\lambda$, there is a BA $B$ of power $\lambda$, such that $B$ does not have onto endomorphisms except the identity.

Of the numerous results about rigid BA's let us mention four. Shelah [S1] proved that for every uncountable cardinal $\lambda$, there is a rigid (that is, without automorphisms except the identity) BA of cardinality $\lambda$. Bonnet [B1], assuming $\mathrm{CH}$, constructed a BA of power continuum, without onto or 1-1 endomorphisms except the identity. Loats [L] and independently Bonnet [B2] generalized Bonnet's construction to $\kappa^{+}$, assuming of course $\kappa^{+}=2^{\kappa}$.

Every BA has some trivial endomorphisms. Let us describe them. Let $B$ be a BA, $a_{1}, \ldots, a_{n} \in B$ and for every $1 \leqslant i<j \leqslant n, a_{i} \cap a_{j}=0 \neq a_{i}$, and $\cup_{i=1}^{n} a_{i}=1$. Let $B \nmid a_{i}$ be the BA that $B$ induces on $\left\{x \mid x \subseteq a_{i}\right\}$, and for every $1 \leqslant i \leqslant n$ let $F_{i}$ be an ultrafilter on $B \uparrow a_{i}$. Let $B^{\prime}$ be the power set of $\{1, \ldots, n\}$, so $B^{\prime}$ is a BA. Let $\sigma \in B^{\prime}$, and let $\pi$ be an endomorphism of $B^{\prime}$ such that for every $\sigma_{1} \subseteq \sigma, \pi\left(\sigma_{1}\right) \supseteq \sigma_{1}$. Let $f: B \rightarrow B$ the unique endomorphism for which: (1) for every $i \notin \sigma$ and for every $x \subseteq a_{i}$ : if $x \in F_{i}$, then $f(x)=\cup\left\{a_{j} \mid j \in \pi(i)\right\}$, and if $x \notin F_{i}$, then $f(x)=0$; (2) for every $i \in \sigma$ and for every $x \subseteq a_{i}$ : if $x \in F_{i}$, then $f(x)=x \cup \cup\left\{a_{j} \mid j \in \pi(i)-\{i\}\right\}$, and if $x \notin F_{i}$, then $f(x)=x$.

Let us call such endomorphisms inevitable.

Shelah [S2] has found this full set of inevitable endomorphisms, and proved that if $\diamond_{\kappa_{1}}$ holds, then there is a BA of power $\kappa_{1}$ with only inevitable endomorphisms.

So at present the following is known.

Presented to the Society, August 17, 1977 under the title On 'strongly' rigid Boolean algebras and cofinalities of BA's; received by the editors November 7, 1977.

AMS (MOS) subject classifications (1970). Primary 02J10; Secondary 02H15.

${ }^{1}$ This constitutes a chapter of the first author's $\mathrm{Ph}$. $\mathrm{D}$. Thesis, prepared at the University of Colorado under the supervision of Professor J. D. Monk. 
(1) ZFC $\Rightarrow$ "For every $\lambda>\aleph_{0}$, there is a BA of power $\lambda$, without onto endomorphisms except the identity."

(2) $\lambda^{+}=2^{\lambda} \Rightarrow$ "There is a BA of power $\lambda^{+}$without onto or 1-1 endomorphisms except the identity."

(3) $\diamond_{N_{1}} \Rightarrow$ "There is a BA without noninevitable endomorphisms."

Whether ZFC is sufficient for (2), and whether, say ZFC $+\mathrm{CH}$ is sufficient for (3) is open. ${ }^{2}$

For BA's with few order preserving functions, see Rubin [Ru] and Shelah [S2].

Finally let us remark about countable BA's. Every countable BA has $2^{\mathrm{k}_{0}}$ automorphisms. See e.g. Monk [M]. Loats in [L] showed also that every countable BA has $2^{\kappa_{0}}$ onto endomorphisms which are not automorphisms.

The proof of Theorem 1.1 combines methods of Shelah [S3] and Reiger [R].

Shelah (unpublished) proved in ZFC that for every uncountable cardinal $\lambda$, there is a rigid dense linear ordering of power $\lambda$. This was done by tagging every element of the linear ordering by a stationary set, more precisely, by an element of the BA $D(\lambda)=P(\lambda) / I(\lambda)$, where $I(\lambda)$ is the ideal of all subsets of $\lambda$, that are disjoint from some closed and unbounded subset of $\lambda$. Reiger [R] used the fact that if $f: X \rightarrow Y$ is a continuous 1-1 mapping, $x \in X$ and there is a 1-1 sequence of order type $\lambda$ converging to $x$, then there is such a sequence converging to $f(x)$. He thus constructed a Stone space of a BA in which the elements are tagged by their cofinalities. In order that each element would have been tagged by a different cofinality he had to assume that the Stone space has cardinality $\aleph_{\alpha}=\alpha$. We noticed that much of Shelah's tagging is preserved under 1-1 continuous functions of the order topology, so we had more tags, and could construct Stone spaces in more cardinalities than Rieger.

\section{The construction.}

Definition. Let $X$ be a topological space and $x \in X$. Then $\operatorname{Cf}(x, X)=$ $\left\{\mu \mid \mu\right.$ is a regular infinite cardinal and there is a sequence $\left\{x_{i} \mid i<\mu\right\}$ in $X$ such that $x=\lim _{i<\mu} x_{i}$ and for every $\alpha<\mu, \lim _{i<\alpha} x_{i}$ exists and is different from $x$ \}. When no confusion might be caused we omit $X$ and write $\operatorname{Cf}(x)$.

Definition. Let $X$ be a topological space, $x \in X$ and $\mu$ be an uncountable regular cardinal; we say that $x$ is $\mu$-special in $X$, if $\mu \in \mathrm{Cf}(x)$, and for every $\left\{x_{i} \mid i<\mu\right\},\left\{y_{i} \mid i<\mu\right\}$ as in the definition of $\operatorname{Cf}(x, X),\left\{\alpha \mid \lim _{i<\alpha} x_{i}=\right.$ $\left.\lim _{i<\alpha} y_{i}\right\}$ is closed and unbounded in $\mu$. From now on let $\lambda$ be a fixed regular uncountable cardinal. If $X$ is a topological space and $x \in X$ is $\lambda$-special, we define $S_{x}^{X}$ to be the element of $D(\lambda)$ gotten in the following way. Let $\left\{x_{i} \mid i<\lambda\right\}$ be as in the definition of $\operatorname{Cf}(x, X)$ and let $S^{\prime}=\{\alpha \mid \lambda \in$ Cf $\left.\left(\lim _{i<\alpha} x_{i}\right)\right\}$. Let $S_{x}^{X}=S^{\prime} / I(\lambda)$. Since $x$ is $\lambda$-special, $S_{x}^{X}$ is independent of the choice of $\left\{x_{i} \mid i<\lambda\right\}$. When no confusion may arise, we write $S_{x}$ instead of

${ }^{2}$ ADDED IN PROOF. New results by Monk and Shelah answer the latter question positively. 
$S_{x}^{X}$. In the sequel we will identify elements of $D(\lambda)$ and their representatives in $P(\lambda)$.

Let $S(B)$ denote the Stone space of the BA $B$.

LEMMA 2.1. $A B A$ B does not have onto endomorphisms except the identity iff $S(B)$ does not have 1-1 continuous functions except the identity.

Proof. Trivial.

Lemma 2.2. If $X$ is a topological space, $f: X \rightarrow X$ is 1-1 and continuous and $x \in X$, then: (a) $\operatorname{Cf}(x) \subseteq \operatorname{Cf}(f(x))$; (b) if $x, f(x)$ are $\lambda$-special, then $S_{x} \subseteq S_{f(x)}$.

Proof. Trivial.

When we refer to a linear ordering as a topological space, we always mean the order topology.

If $X$ is Hausdorff compact totally disconnected space, then $B(X)$ will denote the BA of clopen subsets of $X$; so $X \cong S(B(X))$. If $I$ is a complete linear ordering then $I$ is compact.

If $I$ is a linear ordering, $x \in I$, and $x$ is a left limit (that is, $x$ is not a successor), let $\mathrm{cf}^{-}(x, I)$ be the unique regular cardinal $\mu$ such that there is a strictly increasing sequence of order type $\mu$ converging to $x$; if $x$ is not a left limit, then $\mathrm{cf}^{-}(x)$ is undefined. $\mathrm{cf}^{+}(x, I)$ is defined similarly. It is clear that if $I$ is a complete linear ordering, $x \in I$, then $\operatorname{cf}(x, I)=\left\{\mathrm{cf}^{-}(x, I), \mathrm{cf}^{+}(x, I)\right\}$, where undefined objects are omitted; and $x$ is $\lambda$-special iff either $\mathrm{cf}^{-}(x, I)=$ $\lambda$ and $\mathrm{cf}^{+}(x, I) \neq \lambda$ or is undefined, or $\mathrm{cf}^{+}(x, I)=\lambda$ and $\mathrm{cf}^{-}(x, I) \neq \lambda$ or is undefined.

Let us describe now the aim of our construction for regular cardinals.

LEMMA 2.3. Let I be a linear ordering with the following properties: (1) I is complete; (2) $\mid\{x \mid x \in I$ and $x$ has a successor in $I\} \mid=\lambda$; (3) the set of points of I that have a successor in $I$ is dense in $I$, that is: if the open interval $(y, z)$ is nonempty, then there is $x_{1} \in(y, z)$ such that $x_{1}$ has a successor; (4) for every $x \in I$ either $\lambda \notin \mathrm{Cf}(x)$, or $x$ is $\lambda$-special; (5) there is a dense subset $P \subseteq I$, such that: for every $x \in P, x$ is $\lambda$-special and $S_{x} \neq 0$; for every $x, y \in P$, if $x \neq y$, then $S_{x} \cap S_{y}=0$; and if $x \in I-P$, then either $\lambda \notin \operatorname{Cf}(x)$ or else $S_{x}=0$.

Then: $I$ is Hausdorff compact and totally disconnected, $|B(I)|=\lambda$, and $B(I)$ does not have onto endomorphisms except the identity.

Proof. Let $I$ satisfy conditions (1)-(5). Since $I$ is complete, it is compact. By (3) it is clear that every two distinct elements of $I$ can be separated by a set of the form $V_{x}=\{y \mid y>x\}$ where $x$ has a successor in $I$, and clearly $V_{x}$ is clopen, so $I$ is totally disconnected. It is easy to see that $B(I)$ is the BA generated by $\left\{V_{x} \mid x\right.$ has a successor in $\left.I\right\}$, so $|B(I)|=\lambda$. Suppose now by contradiction, that there is a 1-1 continuous function $h$ from $I$ to $I$, different from the identity. Since $I$ is Hausdorff and $P$ is dense in $I$ there is $x \in P$ such that $h(x) \neq x$. Since by (5) $x$ is $\lambda$-special, $\lambda \in \operatorname{Cf}(x)$; so by Lemma 2.2 (a) 
$\lambda \in \mathrm{Cf}(h(x))$. By (4) $h(x)$ is $\lambda$-special, so by 2.2 (b) $S_{x} \subseteq S_{h(x)}$. However, again by (5), $S_{x} \neq 0$ and $S_{h(x)}$ is either 0 or it is disjoint from $S_{x}$, contradicting the fact that $S_{x} \subseteq S_{h(x)}$. So the identity is the only 1-1 continuous mapping from $I$ to $I$, and by Lemma 2.1, the identity is the only onto endomorphism of $B(I)$. Q.E.D.

Now for every regular cardinal $\lambda$ we are going to construct a linear ordering $I$ as in Lemma 2.3.

Let $\mathbf{Z}$ denote the linear ordering of the integers. If $I$ and $J$ are linear orderings, let $I+J$ denote their sum. If $I$ is a linear ordering and for every $i \in I, K_{i}$ is a linear ordering, let $\sum_{i \in I} K_{i}$ denote the sum of the $K_{i}$ 's over $I$. If $I$ is a linear ordering let $I^{*}$ be the reversed linear ordering. $\lambda$ is considered as a linear ordering, where the ordering relation is $\in$.

We will first describe the construction of $I$, and then list without a proof a series of easy observations, that will lead to the conclusion that $I$ has properties (1)-(5) of Lemma 2.3 .

\section{LEMMA 2.4. The construction of $I$.}

Suppose $S \subseteq \lambda$ is a set of limit ordinals. For every $i \in Z+\lambda$ let us define the linear ordering $J_{i}$ as follows. If $i \in S$ let $J_{i}=1+\lambda^{*}$, where 1 is the linear ordering with exactly one element; if $i \notin S$ let $J_{i}=1$. Let $I_{S}=\sum_{i \in Z+\lambda} J_{i}$.

If $I$ is a linear ordering let $E_{I}$ be the equivalence relation on $I$ defined as follows: $x E_{I} y$ iff there are just finitely many elements between $x$ and $y$. Every equivalence class of $E_{I}$ is convex.

If $x \in I$, let $x / E_{I}$ denote the equivalence class of $x$, and let $I / E_{I}=$ $\left\{x / E_{I} \mid x \in I\right\}$.

ObServation 1. If $A \in I_{S} / E_{I_{S}}$ then $A$ is either of order type $\omega$, or $\omega^{*}$, or $Z$, or $|A|=1$.

We now define $P_{S} \subseteq I_{S}$. It suffices to define $P_{S} \cap A$ for every $A \in I_{S} / E_{I_{S}}$. So let $A \in I_{S} / E_{I_{S}}$ : if $|A|=1$, then $P_{S} \cap A=\varnothing$; otherwise let $B$ be either $\omega$ or $Z$ and $f: B \rightarrow A$ be an onto order preserving or order reversing function; define $P_{S} \cap A=\{f(2 i+1) \mid i \in B\}$. This defines $P_{S}$ up to isomorphism of $\left(I_{S}, P_{S}\right)$.

We now construct linear orderings $I_{n}$ for every $n<\omega$. At the same time we define $P_{n} \subseteq I_{n}$.

Let $\left\{S_{i n} \mid i<\lambda, n<\omega\right\}$ be a family of pairwise disjoint stationary subsets of $\lambda$.

Let $I_{0}=1+I_{\varnothing}+1, P_{0}=P_{\varnothing}$ and let us denote $I_{-1}=\left\{\min \left(I_{0}\right), \max \left(I_{0}\right)\right\}$.

Suppose $I_{n}$ and $P_{n}$ have been defined. Let $f_{n}: P_{n} \rightarrow\left\{S_{i n} \mid i<\lambda\right\}$ be a 1-1 function. For every $x \in P_{n}$, let $K_{x}=I_{f_{n}(x)}+1$, and for every $x \in I_{n}-P_{n}$, let $K_{x}=1$. Let $I_{n+1}=\sum_{x \in I_{n}} K_{x}$. W.1.o.g. we identify $I_{n}$ with a subset of $I_{n+1}$ according to the following embedding $g: g(x)=\max \left(K_{x}\right), x \in I_{n}$. Let $P_{n+1}$ be the subset of $I_{n+1}$ which satisfies: $P_{n+1} \cap I_{n}=\varnothing$ and for every $x \in P_{n}$, $P_{n+1} \cap K_{x}=P_{f_{n}(x)}$. 
Let $I_{\omega}=\cup_{n<\omega} I_{n}$, and $I$ be the completion of $I_{\omega}$ (under Dedekind cuts). Let $P=\cup_{n<\omega} P_{n}$. This concludes the definition of $I$ and $P$.

ObSERVATION 2. (a) $P_{n}$ does not contain limit points. (b) If $x, y \in I_{n}-$ $I_{n-1}$, and $y$ is the successor of $x$, then $x \in P_{n}$ iff $y \notin P_{n}$.

ObSERVATION 3. Let $x \in I_{n}$ and $J \in\left\{I_{k} \mid n<k \leqslant \omega\right\} \cup\{I\}$. Then: (a) If $x \in P_{n}$ and $y$ is the successor of $x$ in $I_{n}$ then $y$ is the successor of $x$ in $J$. (b) If $\lim _{i<\alpha} x_{i}=x$ in $I_{n}$, then $\lim _{i<\alpha} x_{i}=x$ in $J$.

ObSERVATION 4. If $x$ is a limit point $I_{n}$, then $x$ is a limit of a sequence of elements which belong to $P_{n}$.

Proof. By induction on $n$, then distinguish between the following cases: $x \in I_{n}-I_{n-1} ; x \in P_{n-1} ; x \in I_{n-1}-P_{n-1}$.

ObSERvation 5. Let $x \in I_{n}$ and $J \in\left\{I_{k} \mid n<k \leqslant \omega\right\} \cup I$. Then: (a) $x$ is a limit point in $J$; (b) if $x$ is a limit point of $I_{n}$ then $\lambda \notin \operatorname{Cf}(x, J)-\operatorname{Cf}\left(x, I_{n}\right)$.

OBSERVATION 6. (a) $I_{n}$ is a complete linear ordering. (b) If $x \in I-I_{\omega}$, then $\operatorname{cf}^{-}(x, I)=\mathrm{cf}^{+}(x, I)=\aleph_{0}$.

Conclusion 7. I satisfies (1), (2), (3) of Lemma 2.3.

Proof. (1) holds by the definition of $I$. (2) holds by Observations 3(a) and 6. (3) holds by Observations 3(a) and 4.

ObSERvation 8. (a) If $x \in P_{n}$, then $x$ is $\lambda$-special in $I$ and $S_{x}^{I}=f_{n}(x) / I(\lambda)$. (b) If $x \in I_{n}-I_{n-1}-P_{n}$, then either $\lambda \notin \mathrm{Cf}(x, I)$, or $\mathrm{cf}^{+}(x, I)=$ $\lambda, \operatorname{cf}^{-}(x, I)<\lambda$ and $S_{x}^{I}=0$.

Conclusion 9. I satisfies (4) and (5) from Lemma 2.3.

For $\lambda$ regular, Theorem 1.1 now follows from Lemma 2.3 and Conclusions 7 and 9.

Let us denote by $I_{\lambda}$ the linear ordering that we have constructed in 2.4 for $\lambda$. If $\mu$ is a limit cardinal, let $\mu=\Sigma_{i<\kappa} \mu_{i}$ where $\left\{\mu_{i} \mid i<\kappa\right\}$ is a strictly increasing sequence of regular cardinals. Let $I_{\mu}=\left(\sum_{i<\kappa} I_{\mu_{i}}\right)+1$. It is easy to see that $I_{\mu}$ is complete and totally disconnected, $\left|B\left(I_{\mu}\right)\right|=\mu$, and the identity is the only 1-1 continuous mapping from $I_{\mu}$ to $I_{\mu}$.

So Theorem 1.1 is proved.

REMARK. Of course for every $\kappa>\kappa_{0}$, we can construct in the above method a family of $2^{\kappa}$ BA's as in Theorem 1.1, such that there is no homomorphism from one BA in the family onto another.

\section{REFERENCES}

[B1] R. Bonnet, On very strongly rigid Boolean algebras and continuum discrete set condition on Boolean algebras. I, II, Algebra Universalis (submitted).

[B2] _ On very strongly rigid Boolean algebras and continuum discrete set condition on Boolean algebras. III, J. Symbolic Logic (submitted).

[L] J. Loats, On endomorphisms of Boolean algebras and other problems, $\mathrm{Ph}$. D. thesis, Univ. of Colorado, Boulder, 1977.

[M] J. D. Monk, On the automorphism groups of denumerable Boolean algebras, Math. Ann. 216 (1975), 5-10.

[R] L. Rieger, Some remarks on automorphisms of Boolean algebras, Fund. Math. 38 (1951), 209-216. 
[Ru] M. Rubin, A Boolean algebra with few subalgebras and other nice properties, Proc. Amer. Math. Soc. (submitted).

[S1] S. Shelah, Why there are many nonisomorphic models for unsuperstable theories, (Proc. Internat. Congr. Math., Vancouver, B. C., 1974, vol. 1), Canadian Math. Congress, Montreal, 1975, pp. 259-263.

[S2]

Proc. Amer. Math. Soc. (submitted).

[S3] , Private communications.

Department of Mathematics, SOUthern Illinois University, Carbondale, Illinois 62901

Department of Mathematics, University of Colorado, Boulder, Colorado 80309

Current address (James Loats): 1205 Fairfield Drive, Boulder, Colorado 80303

Current address (Matatyahu Rubin): Department of Mathematics, Ben Gurion University of the Negev, Beer Sheva, Israel 